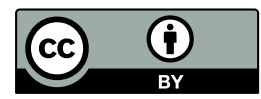

\title{
Análisis de discursos de REDD+ como estrategia equitativa de conservación y uso de la biodiversidad*
}

\section{Analysis of the REDD+ Discourse as an Equitable Conservation and Biodiversity Use Strategy}

\section{Analyse du discours de REDD+ en tant que stratégie équitable de conservation et usage de la biodiversité}

\section{Natalia Orduz Salinas**}

Recibido: 2015-03-10 // Aprobado: 2015-04-20 // Disponible en linea: 2015-06-30

Cómo citar este artículo: Orduz Salinas, N. (2015). Análisis de discursos de REDD+ como estrategia equitativa de conservación y uso de la biodiversidad. Ambiente y Desarrollo, 19(37), 115-130. http:// dx.doi.org/10.11144/Javeriana.ayd19-37.adre

doi:10.11144/Javeriana.ayd19-37.adre

\begin{abstract}
Resumen
El mecanismo de Reducción de Emisiones por Degradación y Deforestación (REDD+) en pocos años ha ganado respaldo institucional y presupuestal del Gobierno colombiano y de organismos multilaterales para su implementación. No obstante, el análisis revela que los actores de interés en el debate público de REDD+ como estrategia equitativa de conservación y uso de la biodiversidad están lejos de lograr un consenso sobre el tema y tienen diferentes niveles de apertura al diálogo. El reconocimiento de miradas culturales distintas (equidad intercultural), de derechos sobre el territorio y la autonomía y de las posibles disyuntivas son asuntos prioritarios para lograr un debate abierto sobre la (in)viabilidad y las condiciones de la estrategia.
\end{abstract}

Palabras clave: REDD+; conservación de la biodiversidad; uso de la biodiversidad; pueblos indígenas; comunidades afrocolombianas; comunidades campesinas

Este artículo es el resultado del trabajo de investigación Análisis de discursos de REDD+ como estrategia equitativa de conservación y uso de la biodiversidad, desarrollado por la autora para optar al título de magistra en Conservación y Uso de la Biodiversidad, dirigido por Diana Lucía Maya Vélez. Recibió aportes y apoyo de Martín Bermúdez y Luis Miguel Renjifo.

** Abogada y magistra en Uso y Conservación de la Biodiversidad, investigadora de la Universidad Nacional de Colombia. Correo electrónico: nataliaorduz@gmail.com. 


\begin{abstract}
The Reducing Emissions from Deforestation and Forest mechanism (REDD+) has received institutional and budget support from the Colombian government and from multilateral organizations for its implementation in just a few years. However, the analysis shows that key actors in the public debate of REDD+ as an equitable conservation and biodiversity use strategy are far from reaching a consensus on the topic, and have different levels of openness to dialogue. Acknowledging different cultural perspectives (intercultural equity) over the rights on territory and autonomy, and on the possible problems, are high priority issues for the achievement of an open debate regarding its (non)viability and the conditions of the strategy.
\end{abstract}

Keywords: REDD+; biodiversity conservation; biodiversity use; indigenous peoples; afroColombian communities; countrymen communities

\title{
Résumé
}

Le mécanisme de Réduction d'émissions par Dégradation et déforestation (REDD+) dans peu d'années a gagné appui institutionnel et budgétaire du Gouvernement colombien et d'organismes multilatéraux pour son implémentation. Néanmoins, l'analyse montre que les acteurs d'intérêts dans le débat public de REDD+ en tant que stratégie équitable de conservation et usage de la biodiversité sont loin d'arriver à un consensus sur le sujet et ils ont différents niveaux d'aperture au dialogue. La reconnaissance de regards culturaux différents (équité interculturelle), de droits sur le territoire et l'autonomie et des possibles disjonctives sont affaires prioritaires pour arriver à un débat ouvert sur l'infaisabilité et les conditions de la stratégie.

Mots clés: REDD+; conservation de la biodiversité; usage de la biodiversité; peuples indigènes; communautés afrocolombiennes; communautés paysannes 


\section{Introducción}

En Colombia el mecanismo de Reducción de Emisiones por Deforestación y Degradación de los bosques (REDD+) goza de gran consideración por parte de distintos actores, así como de disponibilidad de recursos para su regulación e implementación. ${ }^{1}$ El mercado voluntario de REDD+ se ha desplegado desde principios de esta década en Colombia, por ejemplo, a través del programa BIOREDD, operado por USAID y que cuenta con catorce proyectos en la región del Pacífico colombiano. Estos proyectos se rigen por el derecho privado, a través de los acuerdos entre las partes, y muchos tienen cláusulas de confidencialidad. Este análisis, sin embargo, aborda la discusión pública sobre la regulación de REDD+ en Colombia. El Gobierno nacional se encuentra en proceso de formular una estrategia nacional REDD+, con presupuesto y bajo las guías del Fondo Cooperativo del Carbono de los Bosques (FCPF) y de ONUREDD.

La idea de compensar las emisiones evitadas por deforestación en bosques tropicales nació como una medida de cambio climático, en el seno de la Conferencia de las Partes de la Convención Marco de las Naciones Unidas para el Cambio Climático, dado que el Protocolo de Kioto no estimulaba evitar emisiones causadas por deforestación y por degradación de los bosques, aunque estas contribuyen en un $20 \%$ a las emisiones totales de carbono a la atmósfera. Sin embargo, esta propuesta fue cuestionada por centrarse en el carbono y no contemplar los derechos de las comunidades, ni la biodiversidad. En la cop 16 de Cancún ${ }^{2}$ el mecanismo quedó supeditado al cumplimiento de salvaguardas sociales y ambientales $^{3}$ que contemplan, entre otros objetivos, la conservación de la biodiversidad y el respeto de las comunidades locales, de conformidad con las normas nacionales e internacionales que rigen en los países, lo cual incluye los derechos constitucionales de las comunidades al territorio, a la igualdad y a la participación, así como el respeto a los tratados ambientales y de derechos humanos ratificados. ${ }^{4}$

Con lo anterior, REDD+ se ha posicionado como una estrategia que además de combatir el cambio climático, debería contribuir a la conservación de la biodiversidad y generar beneficios a las comunidades. En la mayoría de países tropicales en donde se proyecta este mecanismo, los actores relevantes en el debate son muy diversos entre sí, tanto en su identidad cultural como en la disponibilidad de recursos materiales y simbólicos y el reconocimiento de derechos. En Colombia, los actores rurales y los habitantes de los bosques posiblemente afectadas por REDD+ son al menos tres: pueblos indígenas, comunidades afrocolombianas y campesinos.

1 El Gobierno colombiano se comprometió en la COP 9 del CBD en Bonn con otros 59 países a lograr una deforestación neta de cero en el año 2020. En el 2008 Colombia presentó una propuesta de nota de idea para REDD+ al FCPF que desembolsó 200000 dólares. En el 2011 presentó la quinta propuesta del R-Pp y la FCPF donó 3,6 millones de dólares para la preparación. En el 2013 presentó la séptima versión del R-PP ante el comité de ONU-REDD, el cual autorizó una donación de cuatro millones de dólares (Gómez et al., 2013).

2 La COp definió REDD como el conjunto de cinco medidas: deforestación evitada, degradación evitada, conservación de las reservas forestales de carbono, gestión sostenible de los bosques e incremento de las reservas forestales de carbono (las últimas tres representadas por +). Adicionalmente, estableció que su implementación debía circunscribirse a algunas obligaciones de los Estados, tales como la formulación de la estrategia nacional, un nivel de referencia de emisiones forestales, un sistema de vigilancia forestal y un sistema de información sobre cumplimiento de salvaguardas sociales y ambientales.

3 Las salvaguardas sociales y ambientales son siete y buscan que las medidas REDD+ promuevan y respalden el marco jurídico nacional e internacional, los sistemas de gobernanza forestal nacional transparentes y eficaces, el respeto de los derechos y conocimientos de las comunidades locales, la participación de todos los interesados, la compatibilidad con la conservación de la diversidad biológica y los servicios ambientales, y las medidas para enfrentar riesgos de reversión y para reducir el desplazamiento de emisiones. En Colombia la WwF, en convenio con el Ministerio de Ambiente, desarrolló de manera participativa con comunidades étnicas y campesinas una propuesta preliminar de salvaguardas sociales y ambientales, para lo cual desarrolló una línea base jurídica que contempla los derechos constitucionales de las comunidades rurales.

4 En la cop 9 del свD se estableció que redd no debe ir en contra de los objetivos de esta Convención, antes bien debe proveer beneficios para la biodiversidad, involucrar expertos en biodiversidad, incluyendo conocedores tradicionales y respetar los derechos de los pueblos indígenas y comunidades locales. 
En ese contexto, esta investigación tiene los siguientes objetivos: a) estudiar las diferencias y similitudes de significados que actores relevantes - gobierno nacional, FCPF, pueblos indígenas, comunidades afrocolombianas y campesinos - atribuyen a REDD+ y a su (im)posibilidad de desplegarse como un mecanismo equitativo de conservación de la biodiversidad; b) encontrar los puntos críticos y de consenso sobre las potencialidades, riesgos, alternativas y soluciones que encuentran los actores sobre el mecanismo, como estrategia equitativa de conservación y uso de la biodiversidad; c) analizar los niveles de apertura de cada actor a incluir los significados atribuidos por los demás actores y d) presentar elementos de análisis que posibiliten un debate más abierto e incluyente, también culturalmente, sobre la (in)viabilidad y las condiciones del mecanismo como herramienta equitativa de conservación y uso de la biodiversidad.

\section{Metodología}

Se tomó como marco general el método de teoría fundamentada, que consiste en la construcción de teorías o conceptos a partir de los datos, en vez de otras investigaciones, hipótesis o marcos teóricos existentes. Se trata de un proceso circular de recolección de datos que se analizan, se comparan y se codifican hasta que la nueva información no aporta cambios significativos a los conceptos elaborados (Cuñat, 2007). Los datos teóricos son ideas y conceptos que provinieron de la literatura sobre análisis de discurso y narrativas sobre políticas ambientales, biodiversidad y REDD+; los empíricos, de análisis de discurso de textos de los actores relevantes mencionados arriba. La interpretación que condujo a la conceptualización de los resultados se basó en un ejercicio de hermenéutica diatópica, esta consiste en "un trabajo de interpretación entre dos o más culturas con el objetivo de identificar preocupaciones isomórficas entre ellas y las diferentes respuestas que proporcionan", sobre la base de que ninguna cultura es completa y se complementa con los saberes de las demás (Santos, 2010, p. 53).

La recolección de datos teóricos llevó a la elaboración de categorías de análisis relevantes para todo el estudio. Los datos empíricos provinieron del análisis de discurso, a partir de variables que se explican más adelante. De la recolección mencionada fueron tomados cuatro conceptos que cimentaron el análisis, los cuales se describen a continuación.

\section{Significados}

En una tradición ontológica antiesencialista, en donde la realidad se entiende como socialmente construida, a través de los discursos se filtran, proyectan, legitiman y reproducen algunos significados en vez de otros. Distintos actores luchan por que los significados que les convienen sean posicionados y legitimados en el debate público. Con frecuencia, los conceptos sobre los que existe entendimiento mutuo entre los actores, como "conservación de la biodiversidad", suelen ser etéreos, llenarlos de un contenido concreto, en cambio, suele ser una pugna entre significados (Hajer y Wytske, 2005).

\section{Ideología}

En este análisis, la ideología se entiende como una forma de cognición social, como las creencias compartidas que un grupo tiene y que justifican las prácticas sociales de dicho grupo y su cohesión en torno a la reivindicación de recursos escasos que pueden ser materiales, como dinero o recursos naturales, o simbólicos, como fama o legitimidad. Generalmente, estas creencias compartidas se refieren a características del grupo social como su identidad —nacional, étnica, cultural, política u otra—, su posición en la sociedad, sus intereses, su reproducción, su entorno natural, las razones por las cuales merece acceder a estos recursos escasos para mantener o aumentar su poder y su relación con otros grupos, que en los discursos suele verse representada por una contraposición de argumentos a favor de "nosotros" y en contra de "ellos".

Esta dicotomía se expresa con frecuencia cuando se enfatizan aspectos positivos propios y negativos ajenos y/o se retira el énfasis puesto sobre aspectos negativos propios y positivos ajenos. El discurso 
público, por tanto, es un recurso importante para articular prácticas sociales y moldear el debate público según las creencias compartidas de determinados grupos, por lo que puede ser una importante herramienta de acción conjunta (joint action) de determinados actores sociales con respecto a intereses comunes, pero también una herramienta de dominación de los más poderosos (Van Dijk, 2000).

\section{Disyuntivas en decisiones sobre biodiversidad}

La noción de disyuntivas que subyace a este texto sigue la idea de McShane et al. (2011), según la cual es más realista y efectivo dejar de proyectar y presentar las estrategias de conservación como gana-gana entre muchos intereses, $y$, en cambio, debatirlas sobre el reconocimiento explícito de que estas decisiones son difíciles porque los beneficios y riesgos percibidos por los actores hacen parte de prioridades e intereses distintos, abarcan horizontes de tiempo diferentes y se proyectan en diferentes escalas espaciales; es decir, es inevitable que por algunas ganancias haya que hacer algunas renuncias. Para Visseren-Hamakers et al. (2012), las disyuntivas son contenciosos porque los actores tienen distintas visiones del mundo, por lo que valoran de formas diferentes los riesgos y beneficios, entre los que deben incluirse no solo valores económicos, sino también intrínsecos e indirectos.

\section{Equidades}

Para este texto fueron tenidas en cuenta las tres categorías sobre equidad propuestas por Di Gregorio et al. (2014), a saber: 1) distributiva —basada en distribución de cargas y beneficios—, 2) procedimental - expresada en posibilidades de participación en toma de decisiones- y 3) contextual, anclada en las condiciones de contexto que permiten el ejercicio de las otras formas de equidad. El análisis añadió una nueva categoría a la lista: la equidad intercultural, expresada en el reconocimiento y respeto de otras visiones culturales sobre el mismo aspecto.

De otro lado, los datos empíricos provinieron de las variables de los análisis de discursos. Por su relevancia en el diseño de REDD+, los actores elegidos fueron el Gobierno nacional, que no solo regulará el mecanismo a través de la Estrategia Nacional REDD+, sino que es el garante de los derechos de los ciudadanos, comunidades y pueblos potencialmente afectados por el mecanismo; el Fondo Cooperativo para el Carbono de los Bosques ( establece los procedimientos para su adopción; los pueblos indígenas de la Amazonía, dado que sus territorios tienen alto potencial de albergar proyectos REDD+; las comunidades afrodescendientes del Pacífico, por la misma razón, y otros actores locales, en particular campesinos que también podrían ser afectados directamente por el mecanismo. La selección de pronunciamientos de los actores sobre REDD+ se basó en los siguientes criterios:

- Ser representativos: no son pronunciamientos a título individual, sino que presentan la posición de un colectivo que desempeńa un rol social particular dentro de esta discusión.

- Contener una posición explícita o implícita sobre los aspectos relacionados con la conservación y el uso de la biodiversidad y de equidad del mecanismo.

- Tener un vínculo con el diseño y la implementación de REDD+ en Colombia, lo cual no excluye textos globales o regionales, siempre que tengan relevancia en la construcción doméstica de significados sobre REDD+.

En lo que sigue, las alusiones a los distintos actores hacen referencia exclusivamente a sus pronunciamientos a través de los textos citados en la tabla 1.

El análisis de discurso fue contextual y textual. El análisis contextual detectó, de un lado, la percepción de quien emite el discurso sobre la situación comunicativa (van Dijk, 2000), a través de la identificación de las circunstancias de tiempo, modo y lugar en que se emite el discurso, así como de la audiencia y actores que este reconoce. De otro lado, trazó la red intertextual o universo simbólico y cultural en el que se sumerge el discurso, a través de la identificación de los textos citados y las referencias a otros actores o argumentos de autoridad que cita el emisor. 
Tabla 1. Textos idóneos encontrados y revisados para este análisis

\begin{tabular}{ll}
\hline Actor representado y texto & Consideraciones \\
\hline Gobierno nacional: Bases del & En este texto se encuentran la posición y las medidas que el \\
Plan Nacional de Desarrollo & Gobierno nacional se comprometió a adoptar en el cuatrienio \\
2010-2014 "Prosperidad para & sobre uso y conservación de la biodiversidad y en particular sobre \\
todos" del gobierno de Juan & REDD+. Al escribir este artículo, el Plan 2014-2018 no había \\
Manuel Santos & sido aprobado, sin embargo el anterior representa la posición del \\
& gobierno del presidente Juan Manuel Santos, quien fue reelegido. \\
\hline FCPF: brochure sobre REDD & El Fondo Cooperativo para el Carbono de los Bosques, operado \\
& por el Banco Mundial, quien es su fiduciario, apoya a 36 países a \\
& prepararse para REDD+, entre ellos, Colombia. Sus contribuyentes \\
& son la Unión Europea y varios países de ese continente, Japón y los \\
& Estados Unidos (FCPF, s.f.). El brochure es el texto a través del cual \\
& FCPF explica al público el mecanismo de REDD+ y cómo funciona \\
& el Fondo. Este texto no tiene fecha, pero se puede deducir que es \\
& del 2009 . No incorpora el (+), sin embargo, es el que a la fecha \\
& de redacción de este texto está disponible en la página de internet \\
& oficial del FCPF, por lo cual se puede presumir que para este actor \\
& no ha perdido su vigencia. \\
\hline
\end{tabular}

Organizaciones campesinas y otras de la sociedad civil: "Llamado a la Acción para rechazar REDD+ y las industrias extractivas para enfrentar el capitalismo y defender la vida y los territorios", firmado por 167 organizaciones internacionales y nacionales de la sociedad civil, de veintiséis países convocadas por la Marcha de los Pueblos en el marco de la COP 20 en 2014 en Lima, Perú

Comunidades negras de Pacífico colombiano: "Las comunidades negras del territorio región del Pacífico: su visión de territorio a propósito del cambio climático y el mecanismo REDD+", documento del 2013, de Absalón Suárez en calidad de integrante del Proceso de Comunidades Negras

Pueblos indígenas de la Amazonía colombiana: "Alternativa de REDD+ Indígena: Territorios de Vida Plena para enfriar el planeta." Texto producido por la coordinadora de las Organizaciones Indígenas de la Cuenca Amazónica (Coica, s.f.)
Organizaciones colombianas que suscribieron fueron Censat Agua Viva/Amigos de la Tierra, Amigos del Bosque, Coordinación Campesina del Valle del Cauca y Fundación Beteguma. Es el pronunciamiento encontrado más reciente y completo que respaldaron organizaciones campesinas colombianas: lo firmó la Coordinadora Latinoamericana de Organización del Campo (CLOC) y Vía Campesina, a las que están afiliadas tres organizaciones campesinas colombianas: Coordinador Nacional Agrario, Federación Nacional de Cooperativas Agropecuarias y Federación Nacional Sindical Unitaria Agropecuaria.

El Proceso de Comunidades Negras (PCN) es una organización conformada por 120 organizaciones afrocolombianas étnicoterritoriales de todo el país. Este documento es producto de un proyecto ejecutado en alianza con la organización Patrimonio Natural en el marco del proyecto Incentivos a la Conservación, financiado por la Embajada del Reino de los Países Bajos e implementado con World Wildlife Fund (wwF). El documento enfatiza que no compromete la posición del Fondo Patrimonio Natural, sino únicamente la de su autor en calidad de integrante de PCN.

La Coica es una organización regional, pues de ella hacen parte organizaciones de los distintos países amazónicos, entre ellas la Organización de Pueblos Indígenas de la Amazonía Colombiana (OPIAC). Por su parte, la Organización Nacional Indígena de Colombia (ONIC) respalda las posiciones de COICA sobre REDD+. La Coica, además, en convenio con wwF y con el apoyo del gobierno de Alemania avanza en un proceso para implementar casos pilotos de REDD+ Indígena Amazónico en Perú y Colombia que buscan esclarecer lecciones sobre su diseño e implementación (wwF, 2014). El texto analizado presenta la posición de la Coica sobre REDD+ y su propuesta alternativa. 
El análisis textual, por su parte, se concentró en cuatro aspectos: en primer lugar, el contenido y la forma como los actores perciben la conservación y el uso de la biodiversidad, esto es, la relación entre conservación y uso, causas de pérdida y formas de proteger la biodiversidad. En segundo lugar, la definición que hacen de REDD+ y la atribución de potenciales riesgos, soluciones y alternativas. En tercer lugar, la noción de equidad que cada actor contempla, para lo que se tomaron las categorías expuestas anteriormente. Por último, algunas variables rastrearon la posición ideológica representada en cada texto.

Tabla 2. Variables analizadas en el análisis de discurso

\begin{tabular}{|c|c|c|}
\hline Análisis contextual & nálisis textual & Análisis ideológico \\
\hline $\begin{array}{l}\text { Emisor } \\
\text { Actores reconocidos por el } \\
\text { emisor } \\
\text { Situación } \\
\text { Tipo de texto } \\
\text { Escala (nacional, regional, } \\
\text { local) } \\
\text { Citas y parafraseo de otros } \\
\text { textos, argumentos de } \\
\text { autoridad (análisis inter- } \\
\text { textual) } \\
\text { Recursos disponibles (fuer- } \\
\text { za, territorios y recursos } \\
\text { naturales, conocimiento, } \\
\text { legitimidad, derechos } \\
\text { reconocidos, capital/ } \\
\text { ingresos, fama, recursos } \\
\text { simbólicos) }\end{array}$ & $\begin{array}{l}\text { Biodiversidad: concepto, } \\
\text { conservación, uso, causas } \\
\text { de pérdida, formas de } \\
\text { proteger } \\
\text { REDD+: concepto, potenci- } \\
\text { ales REDD+, riesgos REDD+ } \\
\text { soluciones a riesgos REDD+ } \\
\text { alternativas a REDD+ } \\
\text { Equidades (distributiva, } \\
\text { procedimiental, contextual } \\
\text { e intercultural) } \\
\text { Disyuntivas en REDD+ (ga- } \\
\text { nancias a costa de pérdidas } \\
\text { o riesgos) } \\
\text { Creencias sobre creencias } \\
\text { compartidas (implícito) }\end{array}$ & $\begin{array}{l}\text { Razones por las cuales mi } \\
\text { grupo merece mantener o } \\
\text { aumentar su poder } \\
\text { Elementos reivindicados } \\
\text { para mantener o aumentar } \\
\text { su poder: arreglos, pro- } \\
\text { cesos, actividades, normas } \\
\text { y reglas, recursos (fuerza, } \\
\text { territorios y recursos } \\
\text { naturales, conocimiento, } \\
\text { legitimidad, derechos } \\
\text { reconocidos, capital/ } \\
\text { ingresos, fama, recursos } \\
\text { simbólicos) } \\
\text { Cuadrado ideológico } \\
\text { (énfasis sobre aspectos } \\
\text { positivos propios, retiro } \\
\text { de énfasis sobre aspectos } \\
\text { positivos ajenos, énfasis } \\
\text { sobre aspectos negativos } \\
\text { ajenos, retiro de énfasis } \\
\text { sobre aspectos negativos } \\
\text { propios) }\end{array}$ \\
\hline
\end{tabular}

Fuente: elaboración propia

El ejercicio interpretativo consistió en dos fases: en primer lugar, la búsqueda de similitudes y diferencias de significados sobre REDD+ y su atributo de ser una herramienta equitativa de uso y conservación de la biodiversidad, expuesto en los textos a partir del análisis de discurso de cada uno. En segundo lugar, el estudio buscó develar la apertura de los distintos actores de interés a dialogar con otros. Los indicios de mayor disposición a lograr consensos son los siguientes:

1. Hacer explícitos los fundamentos de la argumentación: el grado de explicación que el texto hace para "ellos" de conceptos claros para "nosotros" (creencias compartidas por los representados en el discurso) es un indicio que razonablemente indica que el emisor intenta tender puentes a otras esquinas ideológicas.

2. Mayor polarización entre "nosotros" y "ellos": se encuentra por vía del análisis del "cuadrado ideológico” explicado arriba.

3. La incorporación de argumentos de equidad procedimental o intercultural: estas dos nciones de equidad son las que muestran una apertura a posiciones distintas. Las otras categorías de 
equidad: contextual y distributiva, por sí solas pueden representar solamente la visión del emisor del discurso.

4. El reconocimiento de disyuntivas: el reconocimiento de únicamente beneficios y no de riesgos o asuntos que tendrán que ceder o ser sacrificados, puede estar invisibilizando los argumentos de otros grupos de interés sobre las cargas que pueden sufrir con el mecanismo.

\section{Resultados}

En primer lugar, se presentan los hallazgos principales sobre los contrastes de significados que los distintos textos otorgan a REDD+ y su atributo de ser una estrategia equitativa de conservación y uso de la biodiversidad. Luego, los diferentes niveles de apertura a buscar consensos en torno al mecanismo.

\section{La conservación y el uso de la biodiversidad como atributo de REDD+ y SU carácter equitativo}

Las diferencias más marcadas de cómo los textos proyectan REDD+ como estrategia de uso y conservación de la biodiversidad recaen sobre tres aspectos. El primero, la manera en que REDD+ se inserta en la comprensión que cada actor tiene de la biodiversidad, de las causas a las que atribuye su pérdida y de la relación entre uso y conservación. El segundo, en cuanto a potencialidades, riesgos, soluciones y alternativas que identifica, y finalmente, las concepciones con relación a las equidades.

Para el Gobierno, la conservación de la biodiversidad es importante para asegurar el crecimiento económico y la competitividad de los sectores y el bienestar. Es de notar que enumera los primeros, pero con respecto a los segundos solamente distingue entre generaciones actuales y generaciones futuras. ${ }^{5}$ Las referencias a la biodiversidad se asocian menos a su valor intrínseco que a su importancia económica, se habla de esta como "capital natural" (DNP, 2011, p. 567) y su uso es mencionado solo en la medida en que resulte competitivo. ${ }^{6} \mathrm{El}$ Gobierno considera que los proyectos que hacen parte de lo que llama "locomotoras" para acelerar el crecimiento económico — término interesante para estudiar en otro lugar - ponen en riesgo la biodiversidad, pero no de manera irremediable: un adecuado ordenamiento territorial y una correcta gestión ambiental son suficientes para hacer ambos propósitos compatibles.

Ese es el papel que desempeña REDD (al que se llama en ocasiones REDD con co-beneficios y a veces REDD+), según el gobierno: una herramienta que no pone en riesgo la competitividad, permite el crecimiento económico bajo en carbono, es esencial para la mitigación del cambio climático y representa oportunidades de financiamiento e incentivos para el país y de desarrollo económico para comunidades. ${ }^{7}$

$5 \quad$ Sobre biodiversidad: "resulta prioritaria su conservación, en pro de asegurar el crecimiento y la competitividad de los sectores turístico, de infraestructura, agropecuario y minero, así como el bienestar de las generaciones actuales y futuras" (DNP, 2011, p. 578).

6 "Con el fin de fortalecer el uso sostenible de la biodiversidad para la competitividad y el crecimiento económico y social, se propenderá por: (1) diseñar e implementar instrumentos para la identificación y valoración de los servicios ecosistémicos y sus vínculos con el bienestar humano; (2) armonizar el marco regulatorio para el acceso a los recursos genéticos; (3) diseñar e implementar una estrategia internacional para la promoción de Colombia como país megadiverso y ambientalmente atractivo; (4) promover la inclusión del principio de corresponsabilidad en los planes sectoriales; y (5) promover la formulación de programas para el desarrollo de tecnologías para la restauración, recuperación, rehabilitación de ecosistemas y uso sostenible de la biodiversidad, en coordinación con el Sistema Nacional de Ciencia, Tecnología e Innovación" (DNP, 2011, p. 579).

$7 \quad$ "Si se sigue con una senda de crecimiento económico con bajas emisiones de carbono, además de los beneficios globales frente a una disminución de GEI, se podrían aprovechar oportunidades de financiación e incentivos que permitirían potencializar las prioridades de desarrollo y no poner en riesgo la competitividad de los sectores colombianos ante una economía global influenciada por estándares de carbono-intensidad. Un ejemplo reconocido es el caso de la estrategia REDD con co-beneficios, la cual va más allá de la deforestación evitada, incluyendo también la posibilidad de compensar las emisiones a 
Su énfasis en equidad es contextual —en clave económica- - y distributiva. Hay un fomento explícito de participación de "sectores y regiones" (DNP, 2011, p. 585), pero no de comunidades y territorios. No hay un reconocimiento a miradas distintas sobre la biodiversidad.

El FCPF no involucra el “+” en su texto, lo que parece dejar en segundo plano los aspectos de biodiversidad y derechos establecidos en Cancún. Sin embargo, promueve una visión del mecanismo que le da importancia a los bosques en pie, lo que tendría varios beneficios, en donde menciona primero varios servicios ecosistémicos preservados, luego inversiones en infraestructura hidráulica y por último la conservación de la biodiversidad y de las culturas. ${ }^{8}$ El Fondo considera que REDD tiene el potencial de generar cambios que impactan de manera positiva "a los países, las provincias, a las personas que viven en los bosques y a las compañías privadas" (FCPF, s.f., p. 9).

El Fondo señala como causa de deforestación el uso ${ }^{9}$ que hacen los habitantes motivados por la pobreza (a quienes llama "dependientes de los bosques" o "pueblos indígenas y otros pobladores" y en algunas ocasiones "comunidades locales"), aunque reconoce su papel en la preservación y por tanto su derecho a participar y ser consultados en el proceso de diseño del mecanismo. Además, resalta que la biodiversidad es importante para la industria farmacéutica. Los grandes proyectos económicos, en cambio, no son mencionados como amenazas a la biodiversidad. Según el texto del Fondo, los riesgos de REDD se previenen con un adecuado diseño. Sus nociones de equidad son, por tanto, contextual - presupone que REDD genera cambios positivos en el contexto ecológico, social y económico-, distributiva — por los beneficios que pueden recibir las comunidades - y procedimental.

El llamado contra REDD+, por parte de organizaciones civiles y campesinas, relaciona este mecanismo con la biodiversidad, pero de forma negativa. No se refiere explícitamente a biodiversidad, sino al territorio y a la naturaleza, para la que exige derechos y en ocasiones llama "Madre Tierra". Así, enlaza el cuidado de la naturaleza con la reivindicación de la autonomía de quienes "viven y son parte de los bosques" (Marcha de la cumbre de los pueblos, 2014, p. 4) como única forma de protegerla y defenderla políticamente.

REDD+ se sitúa en contraposición a esta visión: el texto lo califica como un mecanismo amparado por la lógica de "economía verde", la cual rechaza por considerar que legitima la continuación de las causas del cambio climático, la extracción y consumo de combustibles fósiles, el enriquecimiento de unos a costa del despojo de los pueblos y la mercantilización de la naturaleza. REDD+, de esta manera, perpetúa las condiciones de contexto que tienen resultados injustos en contra de las comunidades.

Hay una disyuntiva entre REDD+ y el tipo de economía que el mecanismo representa según este pronunciamiento, de un lado, y de conservación de la biodiversidad y los derechos de las comunidades, del otro. La única alternativa que se prevé en favor de la biodiversidad y la igualdad es rechazar REDD+, no se exige participación en el diseño del mecanismo. Por lo tanto, el texto reivindica argumentos de equidad contextual e intercultural.

Coica propone una mirada integral y holística de los bosques, y por tanto de REDD+. Así, cuestiona el énfasis en el carbono y propone en cambio una estrategia basada en la protección integral de todo el bosque y de los usos de los pueblos indígenas que durante siglos los han preservado, lo que incluye la satisfacción de sus derechos territoriales, a la autonomía y al consentimiento libre, previo e informado.

través de un manejo sostenible de los bosques, la conservación de la biodiversidad y el incremento de los stocks de carbono en bosques naturales" (DNP, 2011, p. 574).

8 "Además de mitigar el cambio climático, frenar la deforestación y la degradación de los bosques y respaldar un manejo sostenible de dichos bosques ayuda a conservar los recursos hídricos, a evitar las inundaciones, a reducir la escorrentía, a controlar la erosión del suelo, a reducir la sedimentación de los ríos, a proteger la pesca y las inversiones en infraestructura hidroeléctrica, a preservar la biodiversidad, las culturas y las tradiciones" (FCPF, s.f., p. 4).

9 Con respecto a los bosques tropicales: "La tala... la quema... han sido destruidos para obtener provecho económica... para el pastoreo... para el sustento... para sobrevivir" (FCPF, s.f., p. 3). 
Sostiene que como está planteado el mecanismo, "requiere cambios estructurales" (Coica, s.f., p. 2); establece la incompatibilidad entre REDD+ y una economía basada en emisiones de carbono - los créditos de carbono de REDD+ no deben compensar las emisiones de otros sitios- y extracción de recursos naturales.

Coica asimismo identifica numerosos riesgos que acarrea el mecanismo, en especial la pérdida de autonomía sobre el territorio y sus usos. Considera que las salvaguardas son insuficientes, porque no son estructurales, sino formas de mitigación de dańos, y exige los cuatro tipos de equidad. En consecuencia, formula una alternativa REDD+ indígena llamada "Territorios de Vida Plena para enfriar el Planeta", que reta el esquema básico de REDD+.

PCN, por su parte, hace un diagnóstico sobre REDD+ similar al de los pueblos indígenas, en lo que se refiere a su incompatibilidad con grandes proyectos y los numerosos riesgos para las comunidades. Además, coincide en la relación inexorable entre la conservación y el uso de la biodiversidad. En un recuadro azul centrado presenta la ecuación: "Territorio + cultura = Biodiversidad" (Suárez Solís, 2013, p. 9). La diferencia fundamental con el texto indígena, es que no cuestiona el esquema básico de REDD+, sino que plantea las soluciones a los riesgos a través de darle contenido a las salvaguardas.

\section{El nivel de apertura representado en el discurso para dialogar con otras esquinas ideológicas}

El análisis arroja que los discursos más polarizados son el del Gobierno y el de las organizaciones sociales, por vías distintas. El Gobierno, que es junto al FCPF uno de los actores más poderosos por su legitimidad democrática, la disponibilidad de recursos institucionales, técnicos, jurídicos y económicos, basa su argumentación en creencias compartidas trazadas por una lógica economicista, en donde sobreentiende el papel del crecimiento y la competitividad en el bienestar social. Así, desestima a los portadores de otras lógicas y sus argumentos, en particular comunidades locales y sus propios usos, no necesariamente competitivos del territorio. Su red intertextual también excluye estas visiones y actores, a quienes tampoco se propone incluir, pues no reconoce las equidades procedimental e intercultural.

Las organizaciones sociales, al contrario que el Gobierno, no desestiman al "ellos", sino que se concentran en enfatizar todos los aspectos negativos, no solamente de REDD+, sino de todos quienes representan la lógica de "economía verde", lo que incluye a gobiernos, empresas y varias ONG ambientales como WwF y Conservación Internacional. ${ }^{10}$ Su discurso puede ser considerado por esta razón el más ideológico de todos, pues genera con más claridad los criterios de membresía entre "nosotros" (con atributos positivos) y "ellos" (con atributos negativos).

Los argumentos de rechazo a "ellos" son más explícitos y detallados que la descripción de "nosotros", pues el criterio de membresía es el más débil de todos los grupos: se limita a actores atomizados en contra de REDD+, cuyo mayor recurso es el consenso entre actores intercontinentales y diversos en contra del mecanismo. Su red intertextual reposa sobre actores y autores críticos de REDD+.

El discurso del FCPF reposa en la legitimidad que le da ser apoyado por varios Estados. Cuenta con recursos técnicos e institucionales, además de los económicos donados por distintas naciones. Como el del Gobierno, este texto desestima los argumentos de las comunidades locales sobre quiénes son, en el parecer de estas, los verdaderos causantes del cambio climático y la pérdida de los bosques y la

10 Empresas como la petrolera Shell o la minera Río Tinto, de plantaciones forestales, pulpa y papel como Green Resources y Suzano, agroindustriales como Wilmar, Monsanto y Bunge, organismos multilaterales como PNUD o FAO, trasnacionales de la conservación como Wildlife Works, wwF, The Nature Conservancy o Conservación Internacional, empresas consultoras, la banca pública y privada, y muchos gobiernos, elaboran, apoyan y financian proyectos y programas REDD+ y de la agricultura 'climáticamente inteligente'. Estos mecanismos socavan las verdaderas soluciones a la crisis climática, pues son una distracción a los cambios en los modos de producción y consumo y hacia economías y sociedades libres de combustibles fósiles (Marcha de la Cumbre de los Pueblos, 2004, p. 3). 
biodiversidad; en cambio, enfatiza en el papel negativo del uso de las comunidades en la deforestación. Asimismo, considera a las comunidades locales desde una mirada económica, pues su argumento principal es de pobreza y no como sujetos colectivos organizados con reivindicaciones culturales.

A diferencia del texto del Gobierno, resalta la importancia de su participación en el diseño de REDD+ y en las discusiones internacionales sobre el mecanismo. Su red intertextual está en el ámbito de las Naciones Unidas y acuerdos multilaterales ambientales y de cambio climático, pero no menciona explícitamente tratados o declaraciones de derechos humanos.

Finalmente, los dos discursos étnicos pueden ser considerados como los más abiertos para discutir REDD+. Estos actores han logrado acceder con el tiempo a algunos recursos importantes: en algunos casos a territorios colectivos y al reconocimiento de derechos sobre estos y sobre la participación. Cuentan con alta legitimidad global por ser portadores de cosmovisiones y prácticas que preservan el bosque y la biodiversidad. Para ambos, la equidad intercultural es la base de toda la argumentación. Estos dos, además, son los que hacen más explícitos sus argumentos básicos: el texto de PCN explica en detalle la importancia que para las comunidades afrocolombianas del Pacífico tiene el territorio.

El texto indígena, por su parte, detalla la importancia de la mirada holística sobre la vida y la naturaleza, y usa para ello términos occidentales como "servicios ecosistémicos" (Coica, s.f., p. 2). Su reivindicación de participación es muy amplia, pues no solo abarca la consulta previa del Convenio 169 de la OIT, sino también el consentimiento libre, previo e informado, para el que invoca ambos textos: el Convenio 169 y la Declaración de las Naciones Unidas de los Derechos de los Pueblos Indígenas.

Tanto el texto indígena como el texto afro enfatizan en la relación cultural con el territorio para la conservación de los bosques y el papel negativo de sectores económicos como la minería, los hidrocarburos y la agroindustria. También destacan la posición ambivalente del Estado, que promueve políticas ambientales y económicas contradictorias. A diferencia del discurso de las organizaciones de la sociedad civil, no involucran en el "ellos" a las organizaciones ambientales como wwF. Al contrario, trabajan con ellas en relación con el diseńo de salvaguardas, en el caso afro, y de REDD+ alternativo en el caso indígena.

\section{Discusión}

El análisis permite evidenciar que en Colombia los actores están muy lejos de encontrar un consenso, o al menos algunos acuerdos sólidos, sobre REDD+, lo que puede llevar a una implementación del mecanismo conflictiva y poco promisoria para la equidad (comprendida como un conjunto de los cuatro tipos) y el uso y conservación de la biodiversidad. Lo anterior se refleja en cómo algunos actores, sus preocupaciones y sus formas de entender la equidad, el uso y la conservación de la biodiversidad son invisibilizados en los discursos de otros actores. Esto ocurre principalmente en los discursos de los más poderosos: el Gobierno nacional y el FCPF, que no reconocen los argumentos de las comunidades que serían afectadas por el mecanismo, ni sus miradas propias sobre la conservación y el uso de la biodiversidad.

Lo anterior plantea una discusión sobre estrategias de conservación de la biodiversidad: la importancia de asociarlas a la diversidad cultural. En los países en donde se proyecta REDD+ que tienen bosques tropicales existen grupos étnicos diferentes, con usos diversos del territorio, basados en cosmovisiones y prácticas distintas, pero también posicionamientos políticos diferentes. Como se vio, los tres pronunciamientos representativos de indígenas, afrocolombianos y campesinos colombianos tienen diagnósticos en común, pero planteamientos muy distintos de alternativas y soluciones.

Este estudio muestra que el reconocimiento de la diversidad cultural por parte de los actores más poderosos aún es incipiente: el Gobierno en mayor medida y el FCPC algo menos. Ambos excluyen argumentos importantes de las comunidades que serían afectadas, en particular dos: la relación entre REDD+ y los megaproyectos y la importancia de los usos locales y la satisfacción de los derechos de estas 
comunidades para la conservación de los bosques. Estas dos discusiones no son temas privativos de REDD+, sino de muchas otras experiencias sobre uso de recursos naturales y su conservación. Si REDD+ es o no la coyuntura idónea para debatirlos abiertamente es una pregunta. Lo que sí puede afirmarse, es que con estos aspectos sin debatir, la implementación de este y otros mecanismos relacionados con la biodiversidad podrá ser conflictiva.

De otro lado, la posición de los pueblos étnicos —indígena y afrocolombiano- representada en los discursos analizados resultó ser bastante abierta al diálogo sobre el mecanismo, a pesar de una amplia identificación de los riesgos. El motivo de esta apertura debe ser estudiado con mayor detenimiento, pero la alusión constante a las fuentes internacionales de sus derechos puede ser una pista. Varias de las discusiones de aspectos relevantes para ellos, ya fueron resueltas por medio del reconocimiento de derechos, lo que significa que temas que para ellos son no negociables ya están por fuera de la discusión. El planteamiento de REDD+ no puede, por tanto, poner este reconocimiento de derechos en riesgo. Estos actores, en cambio, buscan cómo lograr que el mecanismo, por medio de salvaguardas en el caso afrocolombiano y a través de un planteamiento alternativo en el caso indígena, sirvan para implementar estos derechos.

Finalmente, los motivos de gran polarización del texto que suscribieron las organizaciones campesinas colombianas deben ser estudiados con mayor atención, pero pueden ser el resultado de los dos puntos anteriores: los discursos excluyentes de los actores más poderosos, que conducen a la imposición de medidas en contra de la voluntad de muchos otros actores, y el menor reconocimiento de derechos colectivos y culturales de comunidades campesinas. Si bien su acceso a tierras está protegido por la Constitución, estas comunidades gozan de menos derechos que protejan su relación cultural y colectiva con el territorio, como los pueblos étnicos, tanto a nivel nacional como a nivel global. Existe una declaración universal de pueblos indígenas aprobada, pero una equivalente para campesinos apenas es un proyecto (PNUD, s.f.).

\section{Conclusiones}

Este análisis encontró que la discusión sobre REDD+ entre actores relevantes en Colombia está lejos de lograr un consenso sobre el enunciado "REDD+ como estrategia equitativa de uso y conservación de la biodiversidad". Aunque todos los actores reconocen la importancia de la biodiversidad, atribuyen distintas causas a su degradación y reconocen de manera diferente el papel del uso de las comunidades locales en su preservación. Adicionalmente, comprenden la equidad de manera distinta: las comunidades ponen énfasis en la cultural y procedimental, el Gobierno y el FCPF en la contextual y distributiva, bajo su propio entendimiento de estas.

Ante estos desacuerdos, los actores tienen diferentes niveles de apertura a lograr consensos sobre REDD+. Los más poderosos: el Gobierno y FCPF excluyen argumentos importantes de las comunidades, basadas en su identificación de los riesgos o disyuntivas y de las fuentes normativas que amparan sus derechos. Sobre estos últimos se basa parte de la argumentación de los pueblos étnicos que aceptan el mecanismo, siempre que no vaya en contra de los derechos que han sido reconocidos — al territorio, a la participación, a la integridad cultural - y que en cambio ayude a implementarlos. De otro lado, las comunidades campesinas, representadas en el pronunciamiento en contra de REDD+ firmado por muchas organizaciones de la sociedad civil de distintas partes del mundo, muestran un rechazo absoluto al mecanismo.

Existe entonces una red social global muy crítica, y cuyos argumentos no son abordados por los discursos dominantes. Si bien la destinación de los fondos del FCPF al Gobierno están subordinados a mecanismos de participación con espacio para comunidades y actores locales, una perspectiva excluyente sobre los aspectos mencionados de fondo y visiones limitadas de equidad pueden llevar al cumplimiento de espacios participativos como requisitos, pero no a discusiones con impacto en decisiones de política y proyectos.

De este análisis se desprende que la discusión sobre viabilidad y las condiciones de REDD+ podrá ser abordada de forma más integral cuando: todos los actores reconozcan las disyuntivas identificadas por 
los demás y en particular se debatan, en esta y en otras coyunturas de conservación de la biodiversidad, el papel de proyectos de inversión o extractivos + y el del uso de las comunidades; se hagan explícitos los fundamentos de sus prioridades y su propia argumentación; se aborden los argumentos de equidad desde las distintas perspectivas distributiva, procedimental, contextual e intercultural. Esta última implica el reconocimiento de visiones sobre las relaciones culturales entre uso y conservación de la biodiversidad. Finalmente, el papel de los derechos es imprescindible para permitirles a los actores ingresar al debate sin poner en riesgo aspectos para ellos vitales. El rol del Gobierno, como regulador de la Estrategia, debe actuar conforme a su responsabilidad de garante de estos derechos. 


\section{Referencias}

Adger, W., Benjaminsen, T., Brown, K. y Svarstad, H. (2002). Advancing a Political Ecology of Global Environmental Discourses. Development and Change, 32(4), 681-715.

Coordinadora de las Organizaciones Indígenas de la Cuenca Amazónica (Coica) (s.f.). Alternativa de REDD+ Indígena: Territorios de Vida Plena para enfriar el planeta. Recuperado el 20 de enero del 2015, de http://www.bicusa. org/wp-content/uploads/2014/02/COICA-Alternativa-de-REDD+-Indigena.pdf.

Cuñat Giménez, R. (2007). Aplicación de la teoría fundamentada (grounded theory) al estudio del proceso de creación de empresas. En Asociación Europea de Dirección y Economía de Empresa (2007). Decisiones basadas en el conocimiento y en el papel social de la empresa, XX Congreso anual de Aedem, Palma de Mallorca.

Den Besten, J., Arts, B. y Verjooijen, P. (2014). The Evolution of REDD+: An Analysis of Discursive-Institutional Dynamics. Environmental Science \& Policy, (35), 40-48.

Departamento Nacional Planeación (DNP) 2011. Bases del Plan Nacional de Desarrollo 2010-2014: Prosperidad para todos.

Di Gregorio, M., Brockhaus, M., Cronin, T., Muharrom, E., Santoso, L., Mardiah, S. y Büdenbender, M. (2013). Equity and REDD+ in the Media: A Comparative Analysis of Policy Discourses. Ecology and Society, 18(2). http://dx.doi.org/10.5751/ES-05694-180239

Evans, K., Murphy, L. y Jang, W. (2013). Global versus Local Narratives of RedD: A Case Study from Peru's Amazon. Environmental Science \& Policy, (35), 89-103.

Gómez, R., Hernández, M., Valencia, I. y Guerrero, R. (2013). Informe de seguimiento a la financiación de REDD+ 2009-2012. Bogotá: Fundación Natura.

Gordon, A. y Tam, S. (Eds.) (s.f). FCPF, Brochure. Recuperado el 10 de noviembre del 2014, de https://www. forestcarbonpartnership.org/about-fcpf- 0 .

Griffiths, T. (2009). Seeing REDD? Forests, Climate Change Mitigation and the Rights of Indigenous People and Local Communities. Moreton un Marsch, Reino Unido: Forest Peoples Programme. Recuperado el 2 de febrero del 2015, de http://www.rightsandresources.org/documents/files/doc_923.pdf.

Hajer, M. y Versteeg, W. (2005). A Decade of Discourse Analysis of Environmental Politics: Achievements, Challenges, Perspectives, Journal of Environmental Policy \& Planning, 7(3), 175-184.

Hiraldo, R. y Tanner, T. (2011). Forest Voices: Competing Narratives over REDD+. IDS Bulletin, 42(3), $42-51$.

Ińiguez Rueda, L. (2003). Capítulo III. El análisis del discurso en las ciencias sociales: variedades, tradiciones y práctica. En L. Iñiguez Rueda (Ed.), Análisis del discurso. Manual para las ciencias sociales. Barcelona: Editorial Universidad Oberta Catalunya.

Jerneck, A. (2014). Searching for a Mobilizing Narrative on Climate Change. The Journal of Environment Development, 23(1), 15-40.

Maarten, H. y Versteeg, W. (2005). A Decade of Discourse Analysis of Environmental Politics: Achievements, Challenges, Perspectives. Journal of Environmental Policy \& Planning, 7(3), 175-184.

Manzano, V. (2005). Introducción al análisis del discurso. Recuperado el 5 de mayo del 2014, de http://www.aloj. us.es/vmanzano/docencia/metodos/discurso.pdf

Marcha de la cumbre de los pueblos (2014). Llamado a la acción para rechazar REDD $+y$ las industrias extractivas para enfrentar el capitalismo y defender la vida y los territorios. Lima. Recuperado el 15 de enero del 2014, de http://wrm.org.uy/es/files/2014/11/Llamado-COP-Lima_NoREDD.pdf 
McShane, T., Hirsch, P., Trung, T., Songorwa, A., Kinzig, A., Monteferri, B., Mutekanga, D., Van Thang, H., Dammert, J., Pulgar-Vidal, M. Welch-Devine, M., Brosius, J., Coppolillo, P. y Connor, S. (2011). Hard Choices: Making Trade Offs between Biodiversity Conservation and Human Well Being. Biological Conservation, (144), 966-972.

Movimiento Mundial por los Bosques (World Rainforest Movement) (2015). About WRM. Recuperado el 10 de enero del 2015, de http://wrm.org.uy/about-wrm/

Organización de las Naciones Unidas (2007). Declaración de las Naciones Unidas sobre los Derechos de los Pueblos Indigenas.

Organización Internacional del Trabajo (1989) Convenio 169 de la OIT.

Plan de las Naciones Unidas para el Desarrollo (PNUD) (s.f.). La Declaración de los Derechos de los Campesinos. Recuperado el 12 de marzo del 2015, de http://www.pnud.org.co/hechosdepaz/64/la_declaracion_de_ naciones_unidas.pdf

Proceso de Comunidades Negras (2014). Editorial. Boletín Renacientes, (2). Recuperado el 11 de enero del 2015 , de http://www.renacientes.org/index.php/news-bottom-128/74-ultimas-noticias/1053-boletin-renaciente.

Reátegui Silva, J. (2012). Construcción de REDD+ Indígena Amazónico y Gestión Holística de Territorios de Vida Plena y sus Lecciones. Moyobamba-Perú. Coica. Recuperado el 15 de enero del 2015, de http://www.amazoniaandina.org/sites/default/files/construccion_redd_indigena_amazonico_coica.pdf

REDD-Monitor (2015). About REDD-Monitor. Recuperado el 10 de enero del 2015, de http://www.redd-monitor. org/about/

Robinson, J. (2011). Ethical Pluralism, Pragmatism, and Sustainability in Conservation. Biological Conservation, (144), 958-965.

Ruiz, J. (2009). Análisis sociológico del discurso: métodos y lógicas. Forum: Qualitative Social Research, 10(2). Recuperado el $1^{\circ}$ de abril del 2014, de http://www.qualitative-research.net/index.php/fqs/article/ view/1298/2776.

Santos, B. (2010). Refundación del Estado en América Latina. Perspectivas desde una epistemología del Sur. Bogotá: Siglo del Hombre Editores, Universidad de los Andes, Siglo Veintiuno Editores.

Suárez Solís, J. A. (2013). Las comunidades negras del territorio región del Pacifico: su visión de territorio a propósito del cambio climático y el mecanismo REDD+. Bogotá: Serie de documentos de trabajo: elementos de política del Proyecto Incentivos a la conservación del Fondo Patrimonio Natural, nº 9.

Van Dijk, T. (2000). Ideology and Discourse: A Multidisciplinary Introduction. Barcelona: Pompeu Fabra University.

Visseren-Hamakers, I., Mc Dermott, C., Vijge, M. y Cashore, B. (2012). Trade-Offs, Co-Benefits and Safeguards: Current Debates on the Breadth of ReDD+. Environmental Sustainibility, 4(6), 646-653.

World Wildlife Fund for Nature (WWF) (2014). Alianza Coica y WWF: consolidación e implementación del concepto REDD+ Indígena Amazónico. Recuperado el 2 de enero del 2014, de http://www.wwfca.org/?234950/ Alianza-COICA-y-WWF-consolidacin-e-implementacin-del-concepto-REDD-Indgena-Amaznico 


\section{Siglas}

\begin{tabular}{ll}
\hline CLOC & Coordinadora Latinoamericana de Organizaciones del Campo \\
\hline Coica & $\begin{array}{l}\text { Coordinadora de las Organizaciones Indígenas de la Amazonía } \\
\text { Colombiana }\end{array}$ \\
\hline COP & Conferencia de las Partes \\
\hline FCPF & Fondo Cooperativo para el Carbono de los Bosques \\
\hline ONIC & Organización Nacional Indígena de Colombia \\
\hline ONUREDD & Programa de Naciones Unidas para REDD+ \\
\hline PCN & Proceso de Comunidades Negras \\
\hline PND & Plan Nacional de Desarrollo \\
\hline REDD+ & Reducción de Emisiones por Deforestación y Degradación de Bosques \\
\hline USAID & $\begin{array}{l}\text { Agencia de los Estados Unidos para el Desarrollo Internacional (siglas } \\
\text { en inglés) }\end{array}$ \\
\hline WWW & World Wildlife Fund \\
\hline
\end{tabular}

